Title:

Remote Moving Target Indication Assessment

Author(s):

Gregory H. Canavan, P-DO

Submitted to:

DOE Office of Scientific and Technical Information (OSTI)

DISTRIBUTION OF THIS DOCUMENT IS UNLAMTED
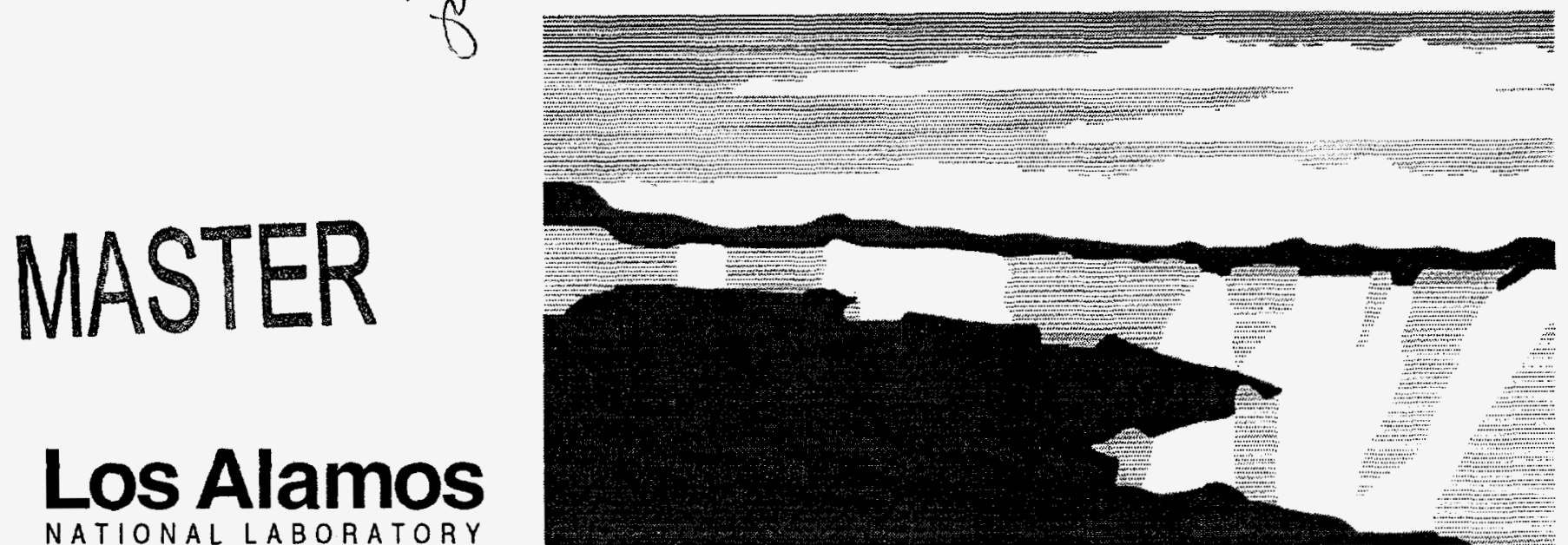

NATIONAL LABORATORY

Los Alarnos National Laboratory, an affirmative action/equal opportunity employer, is operated by the University of California for the U.S. Department of Energy under contract W-7405-ENG-36. By acceptance of this article, the publisher recognizes that the U.S. Government retains a nonexclusive, royaltytree license to publish or reproduce the published form of this contribution, or to allow others to do so, for U.S. Government purposes. The Los Alamos National Laboratory requests that the publisher identify this article as work performed under the auspices of the U.S. Department of Energy. 


\section{DISCLAIMER}

Portions of this document may be illegible in electronic image products. Images are produced from the best available original document. 


\section{DISCLAIMER}

This report was prepared as an account of work sponsored by an agency of the United States Government. Neither the United States Government nor any agency thereof, nor any of their employees, makes any warranty, express or implied, or assumes any legal liability or responsibility for the accuracy, completeness, or usefulness of any information, apparatus, product, or process disclosed, or represents that its use would not infringe privately owned rights. Reference berein to any specific commercial product, process, or service by trade name, trademark, manufacturer, or otherwise does not necessarily constitute or imply its endorsement, recommendation, or favoring by the United States Government or any agency thereof. The views and opinions of authors expressed herein do not necessarily state or reflect those of the United States Government or any agency thereof. 


\title{
Remote Moving Target Indication Assessment
}

\author{
Gregory H. Canavan*
}

\begin{abstract}
This is the final report of a one-year, Laboratory-Directed Research and Development (LDRD) project at the Los Alamos National Laboratory (LANL). The objective of this project was to design and test key components of a sensor to be used on remotely piloted vehicles, aircraft, or satellites for the detection of moving vehicles in cluttered backgrounds. The proposed sensor uses modern large-array focal planes to provide multiple infrared observations of moving targets and capable on-board computers to integrate multiple observations to detect moving targets in background clutter. This combination reduces the size, weight, and cost of the sensor to levels that can be flown on many small unmanned platforms. This effort selected the actual components, integrated them into a test bed, tested the performance of the sensor against realistic generated scenes, and designed a proof-of-concept prototype.
\end{abstract}

\section{Background and Research Objectives}

In previous conflicts, including the Gulf War, the inability to locate and strike mobile targets has been a fundamental limit on U.S. military operations. The precision-guided munitions developed in the last two decades can neutralize targets that remain fixed, but tanks and personnel carriers that move frequently to perform their functions cannot now be detected and localized with enough accuracy and timeliness for precision munition delivery. The fundamental problem is not the munitions, but the sensors that determine where to aim them. This project takes advantage of the very mobility that these targets count on for survivability to locate and track them with the precision and timeliness needed for the effective employment of precision-guided munitions against them. We designed key components of a moving target indication (MTT) sensor that could be deployed on remotely

*Principal investigator, e-mail: gcanavan@lanl.gov 
piloted vehicles, aircraft, or satellites to detect moving vehicles in cluttered backgrounds, resulting in a sensor that could be prototyped rapidly. The resulting sensor is also an important advance for defense and counter-proliferation. It integrates multiple observations to detect targets in the noise of background clutter [1]. That reduces the required signal for detection, which in turn reduces the size, weight, and cost of optical components. This simplification in optics increases the computational burden, but computation rates remain within the capabilities of computers now being flown. They are acceptable even with target detection through straightforward assembly of multiple observations into tracks; they are even lower when more sophisticated neural net or Viturbi algorithms are used to process observations [2].

\section{Importance to LANL's Science and Technology Base and National R\&D Needs}

In addition to the military applications discussed above, there are also non-proliferation and counter-proliferation applications, which involve many of the same considerations, as well as dual-use applications. This approach to remotely screening and cataloging thermal emitters could also be applied to monitoring traffic or land use [3]. Thus, it is an excellent vehicle for technology transfer, because it is an area in which the laboratory has an advantage at the conceptual pre-competitive level, proper facilities, and a logical priority for performing proofof-principle experiments through the brassboard or prototype level. These basic technologies-small satellites and compact, but well-calibrated sensors- - are also essential to laboratory support of environmental monitoring and global climate change. Intended Department of Defense (DoD) customers are the Air Force space and tactical components, other services' tactical components, and intelligence agencies. These sensors would complement high-resolution reconnaissance from complex sensors.

\section{Scientific Approach and Results to Date}

This effort required theory, programming, test, and engineering that produced an analysis for the sensor and a top level discussion of how to modify the sensor and computer for Motorola's Comet satellite remote sensing project. The key feature is that the sensor integrates multiple, low-signal infrared observations to pull moving targets out of noise [1] that reduces the required signal to noise per detection and hence, the size, weight, and cost of optical components. That increases computational burdens, but they remain within those of the flight computer now being tested for Comet [4]. Computation rates are acceptable with track 
assembly of multiple observations and they are even lower with neural net or Viturbi algorithms.

The proposed sensor integrates multiple infrared observations to detect moving targets in background clutter, which would not normally be possible. Small sensors give a large pixel (picture element or footprint) on the ground, the thermal radiation from which can overwhelm that from the target. For example, in the mid-wavelength infrared (MWIR), a typical target would emit about 100 watt/steradian (W/sr). The Earth's radiance in the MWIR is about 1 watt $/ \mathrm{m}^{2}$-sr. A MWIR sensor with a $10-\mathrm{cm}$ entrance aperture at $300-\mathrm{km}$ altitude would have about 10 -m pixels; thus, the background would also be about $(10 \mathrm{~m})^{2} \times 1$ watt/m ${ }^{2}-s r \approx 100$ watt/sr. The single-observation signal-to-noise ratio, $(\mathrm{S} / \mathrm{N}) 1$, would be about unity, which would not support detection at an acceptable false alarm rate. It is possible to overcome this low single-observation signal-to-noise ratio by combining multiple observations of moving targets. To illustrate how that is done, consider the sensor as staring down at a fixed area containing a target. As the target moves, its position shifts from one pixel to another and its image shifts from one detector to another, so it is observed by a succession of detectors. Because fluctuations in the background temperature vary over about the scale of a pixel, the noise level in successive observations are essentially independent. Thus, if the signals from the pixels containing the target are added, the target signal increases as the number of observations, while the noise only increases as the square root of the number of observations. Starting from a single-observation, signal-to-noise ratio of unity, 25 observations would give a combined signal to noise ratio of $\sqrt{25}=5$, which is adequate for detection with an acceptable false alarm ratio. The motion of the platform shifts the images from detector to detector in the trackwise direction, but does not alter the statistics of combining observations from those of the translating frame described above.

More generally, if the sensor makes $M$ independent observations, the $M$-observation signal-to-noise ratio is $(S / N) M \approx \sqrt{M}(S / N) 1$. Thus, the number of independent observations required to achieve a $M$-observation signal to noise ratio $(S / N) M$ is $M \approx[(S / N) M /(S / N) 1]^{2}$. $\mathrm{M}$ varies with target and sensor parameters. For brighter targets, $(\mathrm{S} / \mathrm{N})_{1}$ is higher, which reduces $M$. For more powerful computers, the allowable (S/N)M decreases, which also decreases $M$. Dimmer targets or smaller computers would shift $\mathrm{M}$ in the opposite direction. If the sensor has pixels of diameter $d$ and a transverse search swath $\mathrm{W}$, the number of pixels in the cross track direction in the focal plane array is W/d. For detection, there must also be $M$ pixels in the trackwise direction. The total number of pixels is $\mathrm{MW} / \mathrm{d}$, which is also the number of detectors in the focal plane - a fundamental determinant of performance. There are many possible tracks leading to each of these detectors (and pixels). If only rectilinear tracks of length $M$ are considered, there are about $M$ tracks to each pixel. If rectilinear tracks of 
length $\leq \mathrm{M}$ are considered, there are about $\mathrm{M}^{2}$. For the latter, the evaluation of each track would require about $\mathrm{M}$ operations, so the number of operations for assembling and evaluating all of the tracks leading to a single point scales as $\mathrm{M}^{3}$ and the total number of operations to evaluate all of the possible tracks in the field of view scales as $\mathrm{M}^{3} \mathrm{MW} / \mathrm{d} \alpha \mathrm{M}^{4} \alpha[(\mathrm{S} / \mathrm{N}) \mathrm{M}$ $/(\mathrm{S} / \mathrm{N})]^{8}{ }^{8}$. This result illustrates the importance of powerful on-board computation [to decrease $(\mathrm{S} / \mathrm{N})_{\mathrm{M}}$ ] and good sensors, focal planes, and calibration [to increase $(\mathrm{S} / \mathrm{N})_{1}$ ].

While the discussion above only considered raw computation as a way of reducing signal to noise, there are complementary approaches that take advantage of known characteristics of the area to be searched. By placing a modest map in the sensor computer, it would be possible to ignore areas with low a priori probabilities of targets, e.g., lakes, rivers, mountains, steep slopes, snow fields, and the like. It would appear on the basis of limited investigation that this a priori masking could reduce the required compute rates by about an order of magnitude. The masked regions could be altered adaptively with the transmission of very little information to the platform.

The essential feature of this sensor is observing the area of interest with an array of pixels long enough for a moving target to cover a few dozen of them and adding up the signals along all likely paths to see if the sum is statistically significant. The coverage can come either from repeated observations by a constellation of a few hundred satellites or by using a focal plane array on a single satellite that is sufficiently deep in the trackwise direction. The former could be implemented with linear arrays of detectors, but would require more bandwidth and larger constellations. The latter approach is better suited to a single-satellite proof of principle, but involves more complex optics [1].

Using multiple observations reduces the size, weight, and cost of optical components. This approach has the potential to reduce the optical components to a few-centimeter-aperture camera with modest accuracy and stability requirements, although it will be necessary to evaluate the specific design for tolerances. The required focal planes are available in the form of commercial charge-coupled device (CCD) arrays, which are now available from several vendors in sizes up to a few million detectors [5]. During the course of the study, the PtSi used earlier was replaced with InSb, which has an order of magnitude more efficiency, lower noise, high yield, and large array size in the MWIR. MWIR arrays at the 5002 size now have about the dimensions required for a proof-of-principle sensor focal plane, although they are still about an order of magnitude smaller than required for the ultimate sensor. It will be necessary to provide continuing calibration of planes to within a few percent, because unknown variations of detector responses would degrade the accuracy of combined observations from the ideal values used in estimates above. We used the considerable expertise at Los Alamos in the area of calibration in our design and testing activities. Whether 
commercial arrays can meet the readout speeds required in practice must be determined. However, the required expertise is available within several groups at the Laboratory, where researchers were able to reach speeds adequate for Comet [6].

Computation is a stressing area. A satellite with 10,000 detectors in the cross-track direction with 10 -m pixels (for a $100-\mathrm{km}$ swath) would overfly its pixels about every millisecond and hence generate data at a rate of about 100 megabits per second. That rate is too high to transmit; it must be processed on board. (That is about the rate generated by the sensor on Comet; however, it compresses the data by about a factor of 30 before transmitting it to ground stations.) There are now several flight computers that in units of 3 to 10 central processing units could produce the total computation rates required. The computer on the brilliant pebble produced about 20 million operations per second, which is comparable to the rate above. The Comet R3000 operates at about 30 MIPS. The Rockwell SSPD, which has been space qualified by the Ballistic Missile Defense Office (BMDO) for THAAD, produces about 100 million floating point operations per second, which would provide considerable margin. Each of these computers weighs about a kilogram, so computers do not appear to be mass, power, or cost constraints. Although computation is stressing, it is simplified because the essential operation of track assembly can be computed in a parallel architecture. Thus, computation rates are acceptable even with target detection through track assembly; requirements are even lower when neural net or Viturbi algorithms are used to process the data. Because the data is reduced on the platform, the communication bandwidth required between platforms is modest.

There are a number of tradeoffs between these technologies. In optics, the key parameters are total field of view (for wide swath and long tracks) and small blur circle (for small pixels and high $S / N$ ), which tend to conflict. In the focal plane, the main issues are detection efficiency, uniformity, and cost. Although it appears that commercial PtSi can be purchased cheaply with adequate uniformity, InSb now appears to be available with better efficiency and can be given good calibration. In calibration the key issues are simplicity and uniformity. The techniques developed at Los Alamos for other sensors should be adequate. In computation, the key tradeoffs are availability versus advanced capability and cost. It would appear that paralleling existing computers would suffice, although parallelizing the computations on a single computer would be more efficient for production sensors. Memory and communications could be satisfied with commercial units.

The final product of our LDRD project was the assessment of alternative designs and a rough design for a prototype sensor [7]. 


\section{References}

1. Canavan, G. and E. Teller, "Low-Level Satellites Expand Distributed Remote Sensing," Signal, August 1991, pp. 99-103.

2. Canavan, G. and C. Barrett, "Coverage and Computation from Distributed Surveillance," Los Alamos National Laboratory report LA-12113-MS (1991).

3. Barrett, C., G. Canavan, D. Cooper, R. Joseph, A. Saxman, A. Ledebuhr, I. Lewis, L. Pleasance, W. Scott, and L. Wood, "Applications of Distributed Remote Sensing to Landsat-Like Sensors," Los Alamos National Laboratory document LA-UR-91-3090 (December 1991).

4. Canavan, G. and E. Teller, "Distributed Remote Sensing for Defense and the Environment," Los Alamos National Laboratory document LA-UR-91-1169 (May 1991).

5. Canavan, G. and L. Wood, "Distributed Remote Sensing from Constellations of Small Satellites," A. Zichichi, ed., Proceedings of the Annual World Laboratory Meeting: International Seminar on Nuclear War and Planetary Emergencies (World Scientific, Singapore, 1991).

6. Canavan, G., "Value of Remote Sensing Imagery," Los Alamos National Laboratory report P/AC-95:851 (April 1995: Motorola proprietary).

7. Canavan, G., "'Alternative Architectures," New World Vistas (U.S. Air Force, Air University, in press). 